\title{
Measurements that Matter with Hybrid Model of Estimation
}

\author{
Dragan Stankovski ${ }^{1^{*}}$ \\ ${ }^{1}$ University of Telecommunication and Posts, Sofia, Bulgaria \\ *E-mail of corresponding author: draganstankovski@gmail.com
}

\begin{abstract}
The biggest challenge of delivering projects on time, especially in the field of Telecommunication, is proper planning and correct estimation of the effort. Such estimations while different phases of the development could be extremely thorny and easily can jeopardize final goals and handover on time. Thus in this paper, the proposed approach of Hybrid model estimation is to reduce such unlike stakes and give a clear definition of "how needs to be done" with regular estimations transferred to Story Points.
\end{abstract}

Received 31 October 2021

Accepted 24 November 2022

Published 22 December 2022

Keywords: Estimation, story points, agile, digital transformation, scrum.

\section{Introduction}

The delivery of the project includes different stages and phases between all scrum teams starting from planning, moving across delivery, and finally handing over it with confirmed- and accepted testing results. Each of those phases includes several interactions composed of real measurement of the effort that each team should burn down during the execution in a dedicated frame time. The frame times in agile methodology known as sprints will just visualize the duration of one cycle and the scope that needs to be done as-is committed from the beginning.

To initiate and kick-off one new project means that the full content of the idea should be presented and described as much as possible with the relevant requirements and milestones in business value that should be presented at the end of delivery. When an idea, initiation will be registered, and the execution will start there are a lot of different pieces that need to be predicted as much as possible to avoid jeopardizing projects or in worst-case scenarios not delivering on time. Such risks are tidily connected with the schedule resources and of course the knowledge of the scrum teams who are executing. There are also and additional risks this needs to be taken into consideration, like different dependency from third parties and stakeholders, experience and current knowledge of the project and the product itself, and of course maybe one of the most important is the proper managing of the customer or end clients. The crucial moment in the project lead is a combination of mentioned above that should not be just presented on paper without any measurements or metrics. Nowadays they are huge investigations and a lot of effort to find the right formula and exact estimation productivities. Whit other words are contradictory with the Agile methodology where the scope of work should have its own life cycle and evaluate independently during the different cycles in phases of

(C) The Author(s) 2021. Published by CIEES Association Press. This work is licensed under a Creative Commons Attribution-NonCommercialNoDerivs 4.0 Generic License. execution. The aims and goals in this paper and the next chapters are to provide such correlation and keep both good practices from the agile methodology and clear pattern definition for the exact estimation in the long term of the execution.

\section{Current practice, models of estimations, and major challenges}

When running a project, especially in the field of Telcos most of the managers used the basic definitions of estimation, which it's including the definition of story points to be equal to one personal day of the resources that will execute. By the definition in some small or short initiatives of one project, this can fit the needs and there will be no deviation while delivery, but when the execution is connected to a large number of the squads involving a lot of dependencies between them, and delivering of heavy and difficult pieces of work as a combination of front-end and back-end software development is requiring more detailed and precise planning and definition of risks to get the right estimation and closer value of the story points.

\subsection{The current approach and basic definition of story points}

By the definition and also from the agile perspective to use story points and not working hours has its own advantages like quick estimation or simply said - faster, also it's very easy to measure the velocity of one squad or team that is involved into the work. Calculating the effort of the teams means all additional effort to organize their work such as sending the communication emails, writing the official documentation, using the time to learn or do the knowledge transfer to other team members, and including long hours into different war-rooms when the crisis and defects solving is with the highest priority. Something very important with story points is crucial including the factor of the absence of reliable people like planned vacation, sick leave, etc. Different approaches are defining and include the mapping between story 
points and the working hours and the list is expanding in the several last years, but one of the most common and close to the reality when running projects is the approach of Mike Cohn [1] relations and his relationship is presented in Table 1 .

Table 1. Mike Cohn mapping

\begin{tabular}{cl}
\hline $\begin{array}{c}\text { Story } \\
\text { point }\end{array}$ & \multicolumn{1}{c}{ Mapping to Persons/Days (PD) } \\
\cline { 2 - 3 } $\mathbf{1}$ & Less than 0.5 PD to complete without any complexity \\
$\mathbf{3}$ & $1-2$ PD to complete without any complexity \\
$\mathbf{5}$ & $2-3$ PD to complete without any complexity \\
$\mathbf{8}$ & $4-5$ PD to complete without so much complexity \\
$\mathbf{1 3}$ & $9-10$ PD to complete with some complexity \\
$\mathbf{2 1}$ & $15-20$ PD to complete with more complexity \\
$\mathbf{5 0}$ & Hard to estimate the time, complex for now \\
\hline
\end{tabular}

\subsection{First challenge - Level of estimation}

Usually, when one project is started in the very beginning the management team is expecting to see the effort that should be spent during the execution. Unlikely, in most of the cases, this is only of the level of prediction and the only way to give such numbers is based on pure and previous experience. When it comes to a new type of work and especially including new team members it's breaking the already known predictable type of estimation and it's very difficult to estimate and provide the exact values. A best practice is showing that most of the projects start using T-shirt sizing and implement it on different levels. One of the major challenges and the proposed model is giving a clear definition that T-shirt sizing should happen and will be only valid in the beginning and planning phases while detailed estimation with the exact story points can be presented only during the implementation end execution phases. In this case, there are two ways of estimations named as "top-down" when the estimations are based on the pure planning and they are predicted into the T-shirt shape, and the second one of estimation is defined as "bottom-up" when the real scrum teams provide the correct story points based on the team availability, the complexity of work and wave of execution presented on Figure 1.

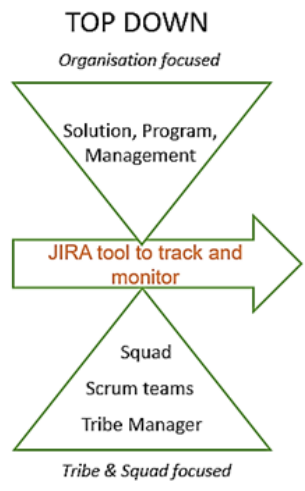

BOTTOM UP

Figure 1. Two-way estimation.

\subsection{The second challenge of Scope and estimation}

The second and very important part of the correct estimation and measurement during the execution of the project is defined with a proper definition of WHAT? need to be measured from the actual work that the team spends in cycles and efforts. In pure agile usually, there is a three-level of hierarchy on the upper level the Epics which clearly describe the intent of the deliverables and bring the real value to the end product in most cases their breakdown to the different User stories that contain dedicated work for each scrum team and the bottom level are presenting the Tasks/sub-tasks that contain real technical work that needs to be done. Besides this real work, there are also additional things involved in the daily work of the team members such as solving issues and bugs/defects, supporting other teams will different tasks, including and innovations, and providing proof of concept (POC) or sometimes named as spikes usually when the team is in the idle state. In the different projects sometimes such additional activities are not taken into the consideration, respectively there is no measurement and this is one of the main reasons to find the real velocity of one team. In the next chapter, the definition of measurements will be directly related to the nature of work and all activities that the scrum teams will execute during the different phases of one project presented in Figura 2.

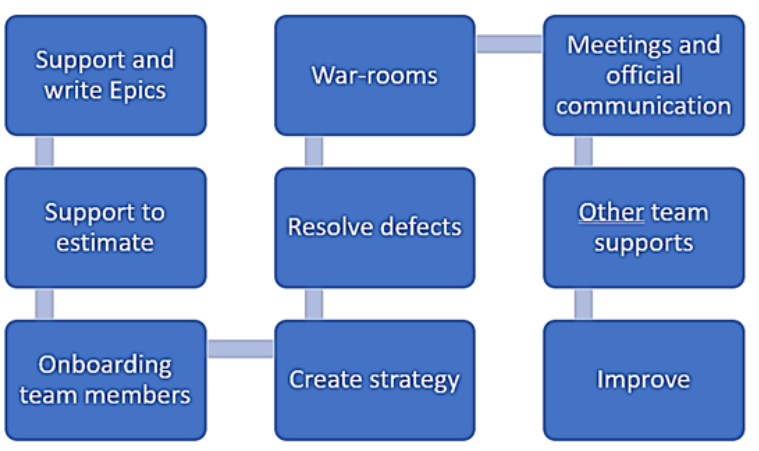

Figure 2. The additional effort is generated in phases of project execution.

\section{Solution and Hybrid model of estimation}

Both challenges mention above for the scope of measurement and level of estimation are leading to the different types of solutions that can be implemented and also modified to provide the best results during delivery. In the next part of this paper, the proposed solution of the hybrid model will give a clear direction what are the exact steps that need to be performed and what is the level of the measurement should be introduced so the project can move forward without any delays or having technical debt on the end of the delivery including and defect code. Have been saying that the first directions to all leading such projects in the field of telcos communication industries are answering to the following:

- What are the issue types that need to be measured?

- What are the order and the level of the measurement?

- What kind of values and complexity are the most appropriate to be used?

- and how we're going to track and improve in every single next life cycle? 
Those four questions named above and presented in Figure 3, actually are going to provide the solution and answer what needs to be done when the scope will arrive into the project backlog.

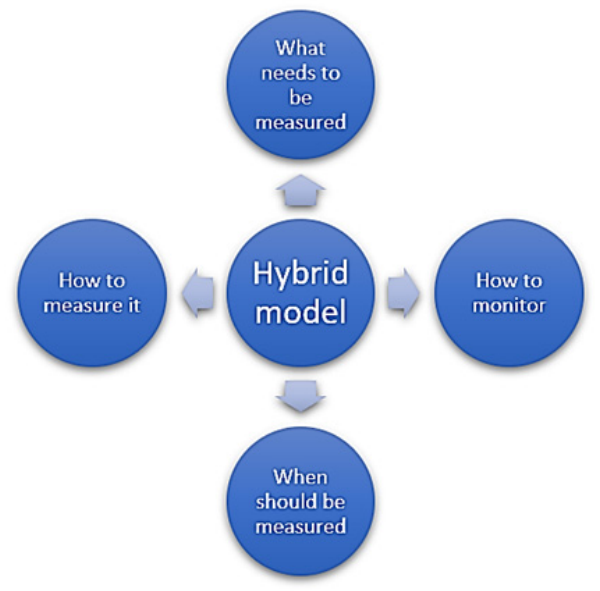

Figure 3. The hybrid model of estimation directives.

\subsection{What should be measured?}

In long-term projects especially in the Telcos in there is always a huge confusion about what needs to be measured. in most of the cases, the squads in the scrum teams except committed work, while the period of execution, are adopting additional tasks mostly interacting as a support to other teams or volunteering completion of additional tasks for improvement as spikes. Such additional things usually are not properly measured, or they're not estimated at all and in most cases, this is causing certain delays and sometimes also the failed delivery content. The first definition of our hybrid model for the estimation is providing the information on what exactly needs to be estimated and point to this statement everything that is requiring $1 / 4$ of the person's daily involvement of one human resource needs to be documented and need to be properly estimated to reduce the waste and gap to the proper estimation. This $1 / 4$ of the regular working hours (in most cases 8 hours per day) are presenting two hours that actually in the worst case can be spent for any additional activities without impacting to deliver on time. Have been saying that any Issue type or type of activities that extend this amount of time should be measured and estimated. The first direction here is that there is no separation between the activities the only trigger to split what needs to be measured and what to be accommodated on a regular daily basis is purely connected to the timing and time consumption. All myths related to spikes bugs support tasks are part of the daily work and they don't need to be estimated is not exactly the right direction and approach, so everything that is more than $25 \%$ should be registered, properly monitored, and of course, reported.

\subsection{How to be measured?}

The second part of this four-wave journey is to provide directions of how things need to be measured. Similar to one of the most famous methods about estimations the better average approach [2] one work can measure the different ways like the optimistic measurements, pessimistic measurement, and most likely measurements with own values. Unlikely into the Telco and their heavy and complex projects, those three factors are not enough, and this is why a new model of estimation is proposed based on the different types and the amount of work that needs to be done. There is always a huge dilemma do project need to be measured in the store points or working hours, not only in this paper there is confirmation that they're always connections and proper definition including the transformation of the working hours with properly included additional working risk factors and the results were going to provide the correct amount of story points. Every single project is unique by its nature not only from the content that should be done and delivered but also unique by the resources who are implementing and executing the project itself. This paper proposes a hybrid model for the estimation taken into consideration are three different factors that were going to provide the total number of the story points used to measure and estimate the real work.

- The first criteria are already presented, and similar criteria as shown in Table 1, but mostly tunned for Telcos needs.

Table 2. Risk factor mapping to PDs

\begin{tabular}{|c|c|c|}
\hline Weight & Description & Parameter \\
\hline 0.5 & $\begin{array}{l}\text { Less than } 0.5 \text { PD to complete without any } \\
\text { complexity }\end{array}$ & \\
\hline 1 & $1 \mathrm{PD}$ to complete without any complexity & \\
\hline 5 & $\begin{array}{l}2-3 \text { PD to complete without any } \\
\text { complexity }\end{array}$ & \\
\hline 7 & $\begin{array}{l}\text { 4-5 PD to complete without so much } \\
\text { complexity }\end{array}$ & PD Effort \\
\hline 11 & $\begin{array}{l}9-10 \mathrm{PD} \text { to complete with some } \\
\text { complexity }\end{array}$ & \\
\hline 21 & $\begin{array}{l}15-20 \text { PD to complete with more } \\
\text { complexity }\end{array}$ & \\
\hline 50 & $\begin{array}{l}\text { Hard to estimate the time, complex for } \\
\text { now }\end{array}$ & \\
\hline
\end{tabular}

- The second and also very important key factor is exactly the level of the knowledge and team skills maturity that actually will give a real start opposite delays caused by lack of time for knowledge transfer and of course learning not only for the product but also and the tools that will be used in the journey.

Figure 4 is presenting the most common practice about the complexity of squad work and the already known including common practice when the scrum teams spend more time working together to have more knowledge about the product. This approach is including not only in their areas as a specialist but also in the product level. This way of working is called I, T, P, or comb-shaped [3]. Also, very important part is to have a deep knowledge of the product, meaning delivering of the same project but for other customers will give an easy start comparing to new project and old client. In this relation. 


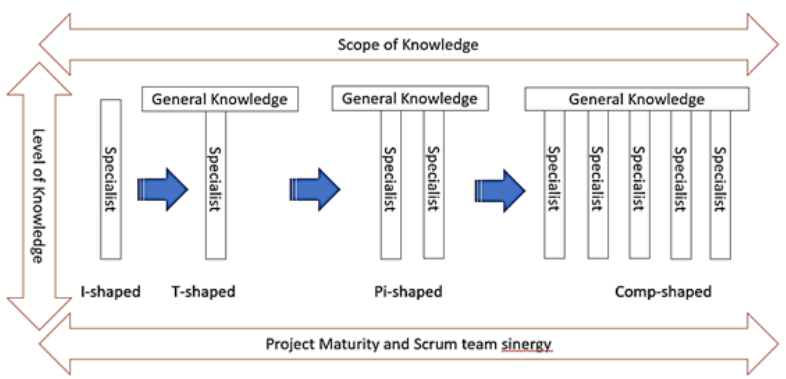

Figure 4. Scrum team's evolution knowledge.

A few things should be taking consideration like the old team members do not know about the project and the product itself, also a second case is when they have some partial knowledge or are fully experts in the fields and the product that will need to be delivered it. Those criteria also are providing the real picture and skill metrics about the resources and usually, it's helping the management team to see where to invest to improve the knowledge of the resources. But clarifying the second factor in this model is presented in Table 3 as the second parameter named "Experience Level".

Table 3. Risk factor mapping to PDs

\begin{tabular}{ccc}
\hline Weight & Description & Parameter \\
\hline 1 & Full experience and known project & \\
2 & Full experience and new project & \\
3 & Partial experience and known project & Experience \\
4 & Partial experience and new project & Level \\
5 & New & \\
\hline
\end{tabular}

The third and very important part to be measured and taking consideration is a very risky and critical factor related to the complexity of the project presented in Table 4.

The complexity itself is the random view and mostly it's driven by the management team. To have a full picture of the complexity of work, need to include and the attitude of the customer, even the third parties dependencies that are always connected part in delivering of every single project. In this manner complexity can be measured on the different levels in this proposal there are six of them to define the work that the squad and the scrum teams need to deliver starting from the very low to the extremely complex.

Table 4. The complexity of project execution

\begin{tabular}{ccc}
\hline Weight & Description & Parameter \\
\hline 1 & Very Low & \\
2 & Low & Complexity \\
3 & Medium & \\
4 & High & \\
5 & Very High & \\
6 & Extremely Hign & \\
\hline
\end{tabular}

The definition of those three factors and criteria that need to be taken into consideration to have a proper calculation of the story points will be presented in the next chapter with the defined equation and results how they need to be calculated so the work that needs to be delivered will have the proper value and there will be minimum possibilities of risks the predicted estimation be different than the executed.

\subsection{When to measure?}

The critical path is to know how and when content needs to be measured this hybrid model is providing two ways of measurements and one single checkpoint in which action is presenting the source of truth and doublecheck of predicted end delivered. In most of the projects, the first measurement of the estimations starts when the content is created. Usually, this is in the hand of the scoping and management team in the solution phase who are establishing the idea or initiating the content that should go to the production. Since this is the very highlevel content

With the high-level content, there's still no clear definition about all requirements or features, not even user stories that need to be delivered and bring value to the customer. Therefore, the most common approach is still having one matrix which it's based on the experience from different projects and just doing the comparison of the level of components from the previous projects. Table 5 presents one matrix we're a T-shirt size is very on high level estimated based on answering few questions and providing to a management team can have a vision about the cost and the timelines of execution the initiatives. An example of such metrics is presented in Table 5 and should be specially created for every single project, up to the needs and his nature plus the uniqueness. This T-shirt measurement it's only on the upper levels or epics and on some SAFe [4] projects could be and features and it is only done before grooming started. Easily can be defined as start estimation. The opposite is the bottom estimation happening on the task level when all details about the requirements are known and confirmed by all stakeholders. This will provide scrum teams easy and based on the guidance in the previous chapter to define story points and commit the content that will go in sprints. Since this is the end level it is defined as a bottom estimation. Usually, the top estimation and the bottom estimation can be double-checked in the middle point, and such projects like the proposed hybrid model the role of the user story level are not for estimated but simply to have this checkpoint and be sure that predicted is the same as executed. When a project starts there is always time for dealing and tuning up especially in some cases when the project is called period for adaptation and mainly it's taking a few springs so the team can find the real measurements tune-up them and afterward use in their planning this statistic usually it's called as a Velocity.

\subsection{How to monitor?}

An important part of this estimation and planning of the work is how-to predict the amount of work that the scrum team should take and commit so they will execute it without any issues during the timelines or milestones. It was mentioned in the previous chapter that the velocity is triggering how many story points spread it through the different tasks, user stories, or epics should be committed in the certain Sprint so the team can work efficiently, and it can be monitored on the different dashboards like the 
progress of the execution and of course the burn down to the due dates. Monitoring of the velocity can be done from different tools, in most cases, it's just the progress and capability of the scrum teams. If the amount of the committed work is completed successfully before the timelines and due date finished it's giving the clear direction that the planning was correct, and accordingly every single deviation like finished but there is remaining work that needs to be placed or very rare cases that work is done but there is still time. These outputs are triggering a consideration that something was wrong during the planning and need attention on how to be fixed or what kind of support and problems the teams are facing so they could not do the proper tasks on time.

\section{Model results and best practices}

In the previous chapter, the presented problems are also explained with a solution that easily can be implemented so for every scrum team to have full visibility and measurements of his work without any impacts and risk for delay or technical depths. Based on the experience in the experimental results during execution of complex project tasks into the telco industry from the period of 2019 till 2020 [5], the suggestions of the hybrid model above provide good and significant results which reduced the technical depth and leftovers of the committed work of the teams from $64 \%$ to only $9 \%$. The following numbers were achieved by strictly implementing this hybrid model and following step by step as is presented below:

- The first step is clear identification of the amount of work that needs to be estimated. As mentioned before in chapter 3.1, everything that it's requiring 1/4 of $\mathrm{PD}$ is an object of estimation. For the current example before the implementation of the hybrid model, the scrum teams were taking work like spikes and working on it without reporting any hours. That leads to struggling and waste of more than two days in some cases which automatically it's leading to jeopardizing of the content, especially in the faster sprints like the UI delivering.

- The most important part is how work needs to be measured so by defining the three-way factors about the expertise, complexity, and personal days - working hours of the resources are implemented in the following equation that the scrum teams can easily predict and calculate exact story points:

$S P=(P D * E L * C) / 0.6$

Example: As a Scrum master the team needs to deliver payment verification with SD secure identification of credit card.

\begin{tabular}{cc}
\hline Weight & Description \\
\hline 1 & Full experience and known project \\
3 & Medium complexity \\
11 & $9-10$ PD to complete with some complexity \\
\hline
\end{tabular}

$S P=(11 * 3 * 1) / 0.6=55$ Story Points
- The final measurement of the velocity is presenting committed work in story points that need to be done and by using the following equation the scrum teams easily can predict next sprint planning and commitments [6]. Depending on the capacity of the team is known that the velocity is not a fixed amount and usually can vary from the different amounts, up to the capacity of the teams. This is including all absences like vacation, sick leaves, etc. Simply less resources less work to be committed.

$\%=\frac{\text { SP end of Sprint }- \text { Commited SP }}{\text { Commited SP }} * 100$

\section{Conclusion}

The proposed hybrid model and the planning of the story points taking into consideration different insights factors that can impact his execution is giving better results about correct planning of the work. During this project, the technical depth was reduced twice. The numbers below are the technical depth that is presented as leftovers moved from one spring to another one. With the current hybrid model of estimation, the achievements are related to proper planning and reduction of the technical depth and fewer requirements that are not executed on time during the different timeframes. For the following period - September 2020 till June 2021 [5] with the same capacity and implementation of the hybrid model presented in this paper the final results increase not only the level of delivery without failures but also and the velocity. The percentage between committed and executed increased to $95 \%$ which is $30 \%$ more compared before implementation of the methodology. Except increasing the velocity of the commitments, the current implementation of the methodology into the development of the software content especially telecommunications companies it's giving a clear definition guide how to do the proper planning and estimation which are leading to easy handling and orchestrating the risk management and reduce the waste. A very important part in reducing the costs and improvements of standards end delivery of quality code that the end client is receiving is the end product to their production.

\section{References}

[1] https://www.mountaingoatsoftware.com/blog/the-mainbenefit-of-story-points.

[2] https://www.netsolutions.com/insights/how-to-estimateprojects-in-agile/.

[3] Conley, Shannon \& Foley, Rider \& Gorman, Michael \& Denham, Jessica \& Coleman, Kevin. (2017). Acquisition of Tshaped expertise: an exploratory study. Social Epistemology. 31. 1-19. 10.1080/02691728.2016.1249435.

[4] https://www.scaledagileframework.com/

[5] Stankovski, D., Bikov, T., Radev, D. (2021), The hybrid model of Agile Transformation in Big Telco corporation, Telfor (under press).

[6] McMillan, B., Atlassian Reporting: Agile Scrum metrics that matter, https://www.cprime.com/resources/blog/atlassianreporting-agile-scrum-metrics-that-matter/ 


\section{Acknowledgements}

The examples and results in this paper are provided from a real project executed in a Telecommunication company shortly mentioned Telco. For the project, purposes are led by Agile methodology a where the human resources are organized into different teams as Scrum teams, and the content that needs to be delivered or the requirements are based on pure Agile and JIRA hierarchy Epic $\rightarrow$ User Story $\rightarrow$ Task. The initial work is scoped and provides the current situation known as "as-is" with dedicated numbers of cycles that will be presented as Interaction in the specific times like Sprints that are with a duration of 2 weeks. The size and measurements are presented as T-shirt and Story points in order to provide orientation for the amount of scoped work and give a minimum valuable product MVP that can be used by end customers navigating through User Interface (UI). 